\title{
Direct teaching on industrial robot without force sensor
}

\author{
Wei Gu ${ }^{1, a}$, Lei Shi ${ }^{1, b}$ and Xincheng Tian ${ }^{1, c}$ \\ ${ }^{1}$ School of Control Science and Engineering, Shandong University, 17923 Jingshi Road, \\ Jinan 250061, People's Republic of China \\ agwei@mail.sdu.edu.cn, 'Pshi@mail.sdu.edu.cn, ctxch@sdu.edu.cn
}

Keywords: Industrial robot, direct teaching, joint torque, position control

Abstract. In order to improve the efficiency of robot teach, this paper presents a direct teaching control strategy for industrial robot with no need for multidimensional force/torque sensor. The kinematics and statics of a specific six-DOF robot are analyzed, and the joints' torque is educed. In the direct teaching process, the servo motors of the robot joints operate in torque/position hybrid mode. Each joint is in dynamic balance status under the corporate impacts of inaccurate motor torque, virtual friction, viscous damping, robot weight, tool weight, and manual drag force. The operator drags the robot/joints easily, and high efficient teach is achieved.

\section{Introduction}

Direct teaching is also known as manual traction teaching, and it is a way that existed early to teach industrial robots. Due to the reaction force of the robot joints, great labor intensity and low teaching precision existed in the early days of direct teaching, then gradually it is replaced by the teach pendant. Even so, the direct teaching has an obvious advantage, namely its efficiency is higher than the teach pendant of several times or even more. This teaching way for some intricate tasks which require a lot of programming has a very important significance, and it makes the direct teaching to become a research focus in the field of the robot high efficiency programming [1].

In recent years, with the continuous development of sensor technology, multiple degrees of freedom (DOF) force / torque sensor is applied more and more widely [2]. By detecting the direction and magnitude of the force / torque which applies to the sensor, the operator drives the tool center point (TCP) to move toward a direction in accordance with the speed. This teaching method is easily operated, but the operator is hard to accurately determine the movement trend of TCP before manipulating the force / torque sensor. Thus the operator has some risk when operating the robot. At the same time, expensive multidimensional force / torque sensor also increases the manufacturing cost of the robot.

In addition to the above-mentioned scheme about direct teaching on the industrial robot, there is also a joint coordinate system direct teaching program - the operator directly moves each joint arm to operate the robot. The most intuitive difference between this method and the above-mentioned method is that it does not need to install the multidimensional force / torque sensor, so it is called direct teaching without force sensor. The basal principle of direct teaching without force sensor is robot's statics balance - the output torque of each joint is just to balance the weight of each arm itself and the end tool, so that the operator can easily move the joint arms. Because the output torque of the robot joint servo motor is detected and controlled based on the motor current, it cannot be strictly accurate. Simple statics balance will result in the balance drift while the robot is not being operated, and cause the sliding of the joint arm. Aiming the direct teaching without force sensor, this paper studies a control strategy, and ultimately achieves stable robot direct teaching with high efficiency.

\section{Establish Robot Coordinate System.}

UR5 is a light general six-DOF robot that Danish Universal Robots Company launched. A simplified UR5 robot model is established, and based on this, the direct teaching is studied. Fig. 1 shows Denavit-Hartenberg (D-H) coordinate system of UR5. 


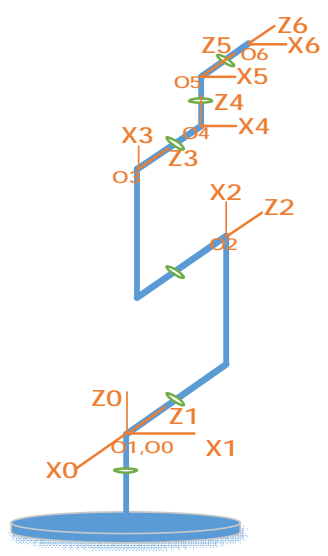

Fig. 1. D-H coordinate system

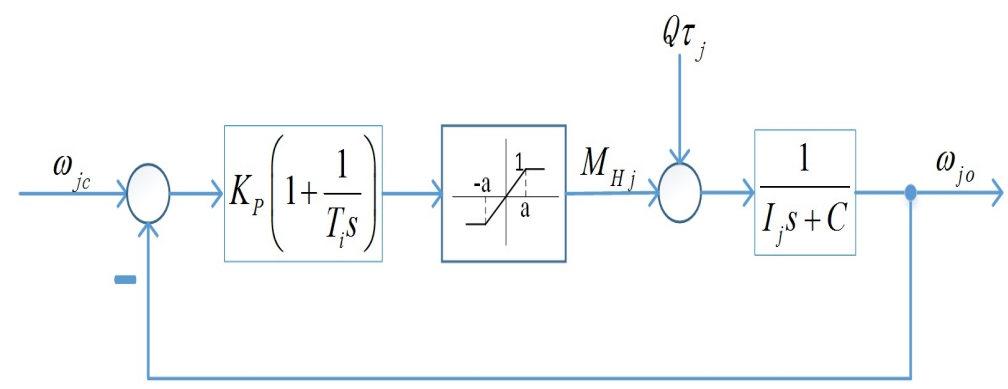

Fig. 2. Structure diagram of direct teaching under dynamic state

\section{Each Joint Torque under Static Balance.}

The robot's manual drawing teaching process is gentler. As there is no violent accelerated and decelerated motion, it is advisable to do its statics analysis rather than dynamics analysis. When teaching, the robot is not in contact with the work piece, the joint torque is mainly used to balance its own arm's weight and the end tool's weight, namely the end operating force of the robot and the operating force of each arm's barycenter are all one dimensional force along $Z_{0}$ axis direction.

By Fig.1, the transformation matrix of each link can be got, and recorded as ${ }_{1}^{0} T,{ }_{2}^{1} T,{ }_{3}^{2} T,{ }_{4}^{3} \mathrm{~T},{ }_{5}^{4} T$, ${ }_{6}^{5} T$. Due to the barycenter of each connecting rod is not coincident with the origin of the corresponding joint coordinate system using D-H method, the barycenter of each link is represented by green circle which are shown in Fig. 1. These transformation matrices from the joint coordinate systems to the matching barycenter are ${ }_{1}^{0} T_{\mathrm{G}},{ }_{2}^{1} T_{G},{ }_{3}^{2} T_{G},{ }_{4}^{3} T_{G},{ }_{5}^{4} T_{\mathrm{G}},{ }_{6}^{5} T_{\mathrm{G}}$ respectively.

Since the gravity of the end tool and each link is parallel to the axis $Z_{0}$, we only care about the position component along $Z$ axis in the posture matrix. We then get the velocity Jacobian matrix of each feature point $-J_{1}, J_{2}, J_{3}, J_{4}, J_{5}, J_{6}$. From Fig. 1 we know $J_{1}=\left[\begin{array}{llllll}0 & 0 & 0 & 0 & 0 & 0\end{array}\right]$. The joint torque vector is denoted as $\tau=\left[\begin{array}{llllll}\tau_{1} & \tau_{2} & \tau_{3} & \tau_{4} & \tau_{5} & \tau_{6}\end{array}\right]^{T}$, and the mass of the connecting rod is marked as $m_{i}(i=1,2,3,4,5,6)$. Similarly, $m_{t}$ represents the mass of the end tool. According to the mapping relationship between the operational force and the joint force under the static equilibrium, we obtain

$$
\begin{aligned}
\tau= & \left(J_{11}^{0} T_{G}\right)^{T} \cdot m_{1} g+\left(J_{22}{ }_{2} T_{G}\right)^{T} \cdot m_{2} g+\left(J_{33}^{2} T_{G}\right)^{T} \cdot m_{3} g+\left(J_{44}^{3} T_{G}\right)^{T} \cdot m_{4} g \\
& +\left(J_{5}{ }_{5}^{4} T_{G}\right)^{T} \cdot m_{5} g+\left(J_{66} T_{G}\right)^{T} \cdot\left(m_{6}+m_{t}\right) g \\
= & \left(J_{22} T_{G} T^{T} \cdot m_{2} g+\left(J_{3}{ }_{3}^{2} T_{G}\right)^{T} \cdot m_{3} g+\left(J_{44}^{3} T_{G}\right)^{T} \cdot m_{4} g+\left(J_{5}{ }_{5}^{4} T_{G}\right)^{T} \cdot m_{5} g+\left(J_{66} T_{G}\right)^{T} \cdot\left(m_{6}+m_{t}\right) g\right.
\end{aligned}
$$

Supposing $T_{i}=J_{i}{ }_{i}^{i-1} T_{G}$, we rewrite Eq. 1 as

$$
\tau=T_{2}^{T} \cdot m_{2} g+T_{3}^{T} \cdot m_{3} g+T_{4}^{T} \cdot m_{4} g+T_{5}^{T} \cdot m_{5} g+T_{6}^{T} \cdot\left(m_{6}+m_{t}\right) g \quad .
$$

Each joint torque is

$$
\tau_{\mathrm{j}}=\sum_{i=2}^{6} T_{i j} \cdot m_{i} g+T_{6 j} \cdot m_{t} g \quad,(j=1,2,3,4,5,6)
$$




\section{Direct Teaching Control Strategy.}

Torque control of the servo motors is realized by the current loop of the servo control systems [4]. However, the constant relationship between the motor output torque and the corresponding current is easily affected by various practical factors (core material saturation, change of reluctance torque, armature temperature, etc.).This change will result in greater accuracy deviation in the torque control ( $5 \%$ to 20\%; the deviation of lighter load is smaller, generally less than 5\%) [5]. The basic control mode of direct teaching without force sensor is joint torque control. The inaccuracy of servo motor torque control has a negative impact on the direct teaching, and the following two aspects are analyzed.

Torque Balance under Dynamic State. The feature of this state is smaller external operating force can drive each joint arm to move in a specific direction and make them stop quickly. To further study this problem, we need to define some other variables. Presume the angle vector of each joint is $q=\left[\begin{array}{llllll}\theta_{1} & \theta_{2} & \theta_{3} & \theta_{4} & \theta_{5} & \theta_{6}\end{array}\right]^{T}$. The instruction torque from the controller to the servo motor is $M_{D j}(q)$ which is transmitted to the joint by reducer, and the actual driving torque is $M_{Q j}(q)$. The torque is $M_{T j}(q)$ that generated by the gravity of each arm and the end tool maps to each joint. The joint damping torque is $M_{F j}(\omega)$. The moment of inertia of the rotating part of each joint is $I_{j}(q)$. The torque acting on each joint is $M_{H j}(t) . M_{T j}(q)$ is exactly the joint torque under static balance; that is

$$
M_{T j}(q)=\tau_{j}(q)
$$

Each joint instruction torque is supposed to equal each joint torque, that is

$$
M_{D j}(q)=\tau_{j}(q) .
$$

However, the actual driving torque of each joint has a certain inaccuracy:

$$
M_{Q j}(q)=M_{D j}(q) \cdot(1+Q),
$$

where $Q \sim N\left(0,0.1^{2}\right)$.The joint damping torque is mainly from the reducers, which is simplified as linear viscous damping, namely

$$
M_{F j}(\omega)=C \omega,
$$

where $C$ is damping coefficient after conversion, its unit is $N \cdot \mathrm{m} / \mathrm{s}^{-1}$. Assume that the robot has only one joint at the rotation under the action of external force. If the angular speed is $\omega$, the kinematics equation for this joint is

$$
I_{j} \frac{d \omega}{d t}+M_{F j}+M_{T j}=M_{Q j}+M_{H j} .
$$

Substituting Eq. 4, Eq. 5, Eq. 6, and Eq. 7 into Eq. 8, we get

$$
I_{j} \frac{d \omega}{d t}+C \omega=Q \tau_{j}+M_{H j}
$$

Supposing $\omega(0)=0$ and $\frac{d \omega}{d t}(0)=0$, by doing Laplace transforms of the Eq. 8, we obtain

$$
\left(I_{j} s+C\right) \omega(s)=Q \tau_{j}(s)+M_{H j}(s) .
$$

The human brain is simplified as a simple PI controller. $M_{H j}$ is the torque that the person exerts on a certain joint. The joint angular speed $\omega$ is the controlled object. The structure diagram of direct teaching under dynamic state is shown in Fig. 2. 
We take a set of typical values in the process of direct teaching of UR5 robot: the maximum external operating torque is $a=50 \mathrm{~N} \cdot \mathrm{m}$, the moment of inertia of the rotating part of each joint is $I_{j}=1.25 \mathrm{~kg} \cdot \mathrm{m}^{2}$, the joint damping torque coefficient is $C=5 \mathrm{~N} \cdot \mathrm{m} / \mathrm{s}^{-1}$, the joint torque is a function of the joint angles which is abstracted as $\tau_{j}=20 N \cdot m \times \sin \left(\int_{0}^{t} \omega d t\right)$, and the torque error coefficient $Q$ of each joint can be considered as a constant value, namely $Q=0.05$. The time domain curves of the instruction speed $\omega_{j c}$, the output speed $\omega_{j o}$ and the manual torque $M_{H j}$ are shown in Fig. 3.

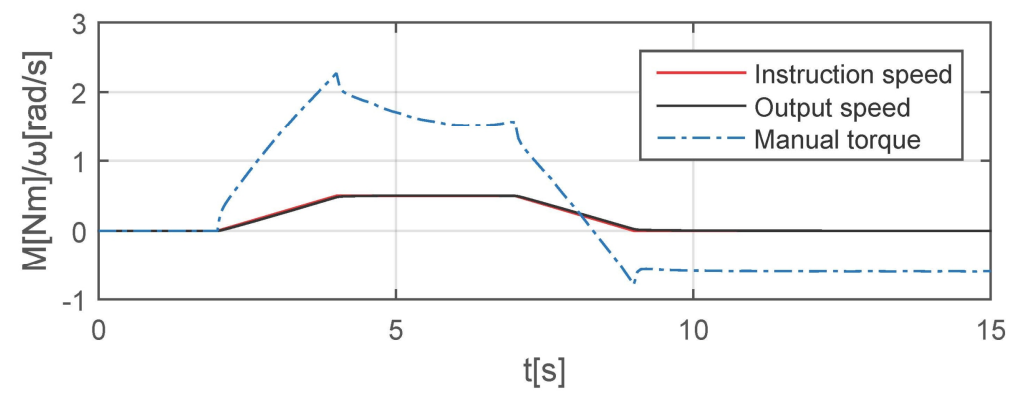

Fig. 3. Simulation result of the time domain curves under dynamic state

As we can see from Figs. 2 and 3, the external operating force can easily control the angular speed of each joint arm, and further control their position. Thus, under the dynamic state, each joint instruction torque only needs to meet Eq. 5 so that the external force can easily achieve direct teaching.

Torque Balance under Static State. Static state refers to the process of direct teaching, in which the robot remains still under the condition of no external force. This state is characterized by when the robot is static and the external force doesn't appear in this progress, the position of the robot's arms will not appear to drift; after the action of a smaller force, the robot joint arms will move in the specified direction. If the control strategy of the dynamic state is adopted, and we see from Eq. 9, in the absence of external operating force, each arm may produce the position drift due to the presence of the joint torque error $Q \tau_{j}$. Therefore, this control strategy can't meet the requirements of direct teaching in a static state.

The position control mode is a working mode of the servo systems, which is used in the normal work of the robot. When the positional instruction that the servo motor receives is not changed, the servo motor remains stationary, and the output torque will automatically adjust and keep balance with the rest of the torque (constant or variable) that applies to the joint. Under the right conditions, to make a joint servo motor enter the position control mode will avoid the occurrence of position drift; similarly, under the appropriate conditions the operator makes it exit the position control mode and re-enter the torque control mode for teaching. The force that used to balance the joint torque error under the position control mode is called virtual static friction force. Next, we will study the two modes of entering and exiting the position control.

First, we consider the model of entering the position control. A needed condition for the existence of static friction force is two objects contact with each other and remain relatively stationary, so we regard the joint speed equals zero as a necessary condition for entering the position control mode. However, when a certain joint requires to reciprocally fine-tuning, frequently entering the position control mode will bring greater inconvenience to teach. We set a smaller joint speed window which contains zero, for example $[-0.05 \mathrm{rad} / \mathrm{s}, 0.05 \mathrm{rad} / \mathrm{s}]$. When a joint speed for a period of time (for example, 2 seconds) is inside this window, the servo motor is switched to the position control mode. The conversion condition is expressed as the following formula:

$$
\forall t \in\left[t_{\text {now }}-2, t_{\text {now }}\right], \omega(t) \in[-0.05 \mathrm{rad} / \mathrm{s}, 0.05 \mathrm{rad} / \mathrm{s}] \text {. }
$$


Second, we discuss the situation of exiting the position control mode. The servo motors work in the position control mode, and output the current torque signal. When the robot is in the static state or the slow teaching state, the actual output torque of each joint under the position control mode is

$$
M_{A j}(q)=\tau_{j}(q) .
$$

Assume that this joint is not affected by the external force. The output torque of the joint is denoted as $M_{B j}$, which is got by transforming the current torque of the servo systems, then it should meet the following constraint:

$$
\frac{M_{B j}(t)}{M_{A j}(q)}=\frac{1}{1+Q} \in(0.9,1.1)
$$

When this joint is operated by more obvious external force, $M_{B j}$ will no longer satisfy Eq. 12 . At this time, the joint servo can exit the position control mode, return to the torque control mode. The transition condition is expressed by the following formula:

$$
\frac{M_{B j}(t)}{M_{A j}(q)} \notin(0.9,1.1) \text {. }
$$

\section{Conclusions}

This paper studies the direct teaching without force sensor, and obtains the control methods of the robot's joints under the dynamic state and static state and the conversion conditions of two states. The joint is in a torque control mode (refer with: Eq. 5) when it is moving; when the joint speed satisfies Eq. 11 , the joint is switched to the position control mode (static state). This control strategy can realize the direct teaching without force senor, which can improve the teaching efficiency of the industrial robot.

In this paper, the joint statics' calculation method based on the simplified model is not practical, because the control strategy depends on the statics' calculation of the joint. Therefore, more accurate robot models are needed in the actual engineering, including the mass of each arm and the position of each barycenter. And more precise torque control is a prerequisite for the smooth teaching. When the error of the torque control is relatively large and the servo systems quit the position control mode, the greater virtual static friction force will make the external force become larger.

\section{Acknowledgments}

The authors gratefully acknowledge the research funding by National High-Tech Research and Development Program of China (863 Program) under Grant No. 2015AA034404.

\section{References}

[1] Zhanxian Li, Jie Chen, Zhijie Yang, Liya Zhao. Design and Optimization of the Gravity Balancing for the Joint-type Robot Teaching Mechanism [J].Mechanical Engineer, 2010, 9:30-32. (In Chinese)

[2] Kezhuan Zhao, Zeyu Xu. Design and study of a new type of six-dimensions force sensor for Industrial robot [J].Transducer and Microsystem Technologies, 2015, 34(5):5-7. (In Chinese)

[3] John J. Craig. Introduction to Robotics: Mechanics and Control (3rd Edition) [M]. New Jersey: Prentice Hall, 2004.

[4] Ping Qian. Servo System (2rd Edition) [M]. Beijing: China Machine Press, 2011. (In Chinese) 
[5] Jing Xue, Dr. Guido, Stoeppler, Dr. Franz Bauer. Measures to improve the torque control precision of permanent magnet synchronous motor [J]. Power Electronics, 2008, 01:22-25. (In Chinese) 